\title{
Echocardiographic Diagnosis of Transposition of the Great Arteries Associated with Anomalous Pulmonary Venous Connection
}

\author{
Lilian Maria Lopes, Gláucia Maria Penha Tavares, Fred Luiz Mailho, Vicente de Paulo Cavalcante de Almeida, \\ José Armando Mangione \\ São Paulo, SP - Brazil
}

We report 2 cases of transposition of the great arteries associated with anomalous pulmonary venous connection emphasizing the clinical findings, the diagnosis, and the evolution of the association. One of the patients had the anomalous pulmonary venous connection in its total infradiaphragmatic form, in the portal system, and the other patient had a partial form, in which an anomalous connection of the left superior lobar vein with the innominate vein existed. At the time of hospital admission, the patients had cyanosis and respiratory distress with clinical findings suggesting transposition of the great arteries. The diagnosis in 1 of the cases, in which the anomalous connection was partial, was established only with echocardiography, without invasive procedures that would represent risk for the patient; in the other case, in which the anomalous connection was total, the malformation was only evidenced with catheterization. The patients underwent surgery for anatomical correction of the heart disease. Only 1 patient had a good outcome.

The association of transposition of the great arteries and anomalous pulmonary venous connection is very rare ${ }^{1,2}$. With few exceptions, the cases reported in the international literature result from postmortem studies ${ }^{3}$ and, many times, are part of very complex syndromes, usually with death occurring a few hours or days after birth.

Therefore, we considered the report of these cases of clinical interest, due to the rarity of the association and the contribution of the sensitivity of the echocardiogram as a preoperative method of diagnosis.

Real e Benemérita Sociedade Portuguesa de Beneficência

Mailing address: Lilian M. Lopes - Rua Batista Caetano, 79/111 - 04108-130 São Paulo, SP, Brazil - e-mail: ecokid@ecokid.com.br

English version by Stela Maris C. e Gandour

\section{Case Reports}

Case 1 - The patient was a 22-day-old male infant with a history of mild cyanosis since his birth. On physical examination at the time of admission, the infant was moderately tachydyspneic, and on heart auscultation, an increased intensity of the $2^{\text {nd }}$ heart sound predominated.

The echocardiogram showed transposition of the great arteries, ventricular septal defect of $3 \mathrm{~mm}$ in diameter, atrial septal defect of the oval foramen type with a diameter of $4 \mathrm{~mm}$, and pulmonary flow with a pattern of pulmonary hypertension. The great dilation of the right chambers was noteworthy (fig. 1A), but an association with anomalous connection was not suspected. The echocardiography also showed a single coronary ostium, which was confirmed on cardiac catheterization.

The patient underwent cardiac catheterization for assessment of the coronary arteries and performance of atrioseptostomy. During the procedure, the echocardiographicfindings were confirmed, and an anomalous infradiaphragmatic connection of the pulmonary veins of the nonobstructive type was shown (fig. 2).

The surgical procedure chosen was the anatomical correction of the transposition of the great arteries, implantation of the anomalous pulmonary vein in the left atrium, ventriculoseptoplasty, and resection of a small muscular band from the right ventricular outflow tract that caused a mild subaortic stenosis (intraoperative finding). The patient evolved satisfactorily, and was discharged from the hospital after 2 weeks.

Case 2 - The patient was a 5-month-old male child weighing $4.029 \mathrm{~kg}$, who, on physical examination, was moderately cyanotic, malnourished, and tachydyspneic. On cardiac auscultation, a systolic murmur $(3+/ 4)$ predominated on the medium-to-low portion of the left sternal margin, and increased intensity of the $2{ }^{\text {nd }}$ cardiac sound in the pulmonary area was observed.

Echocardiography showed transposition of the great 


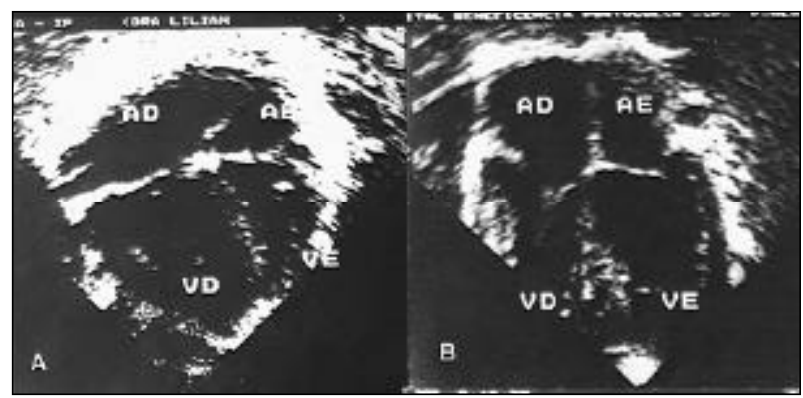

Fig. 1 - Four-chamber view. A) Observe the severe dilation of the right chambers in case 1 , which was associated with the total form of anomalous pulmonary venous connection; B) Observe the subtle predominance of the right atrium in this echocardiographic view in case 2 , which was associated with the partial form of anomalous pulmonary venous connection.

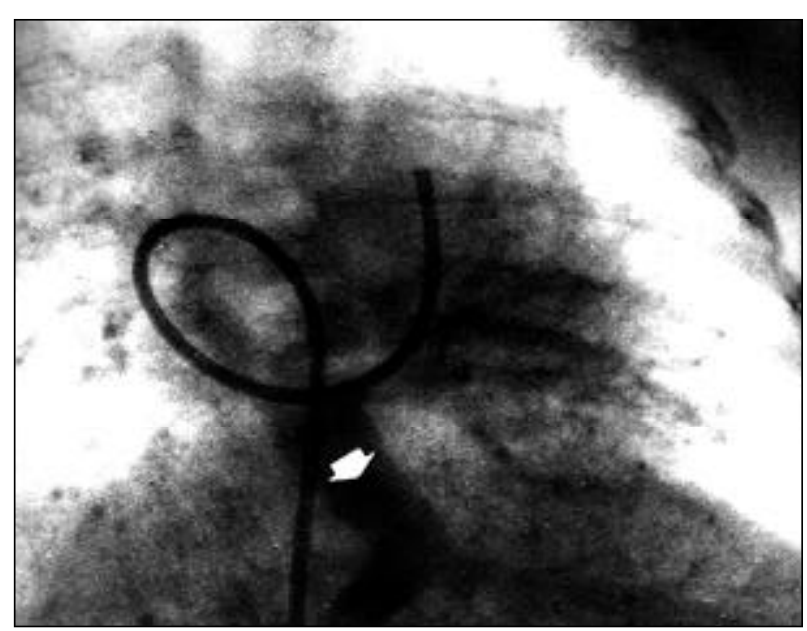

Fig. 2 - Catheterization, case 1. Angiographic diagnosis of the infradiaphragmatic anomalous pulmonary venous connection. Contrasted pulmonary venous return and anomalous vein heading down to the infradiaphragmatic region (arrow).

arteries (fig. 3), multiple ventricular septal defects, signs of pulmonary hypertension, mild dilation of the right chambers (fig. 1B), and also the presence of partial anomalous pulmonary venous connection, in which, the pulmonary vein of the left superior lobe drained into a vertical vein that followed the innominate vein and superior vena cava (fig. 4).

The child underwent surgery with no complementary hemodynamic investigation, and, during surgery, the echocardiographic diagnosis was confirmed. Anatomic correction of the great arteries and correction of the other defects was performed. The postoperative evolution was unsatisfactory, and the child evolved to death on the $3^{\text {rd }}$ postoperative day due to renal failure, which was followed by multisystem organ failure.

\section{Discussion}

Transposition of the great arteries associated with anomalous pulmonary venous connection is rare ${ }^{1-5}$. However, the association of these 2 defects may attenuate the

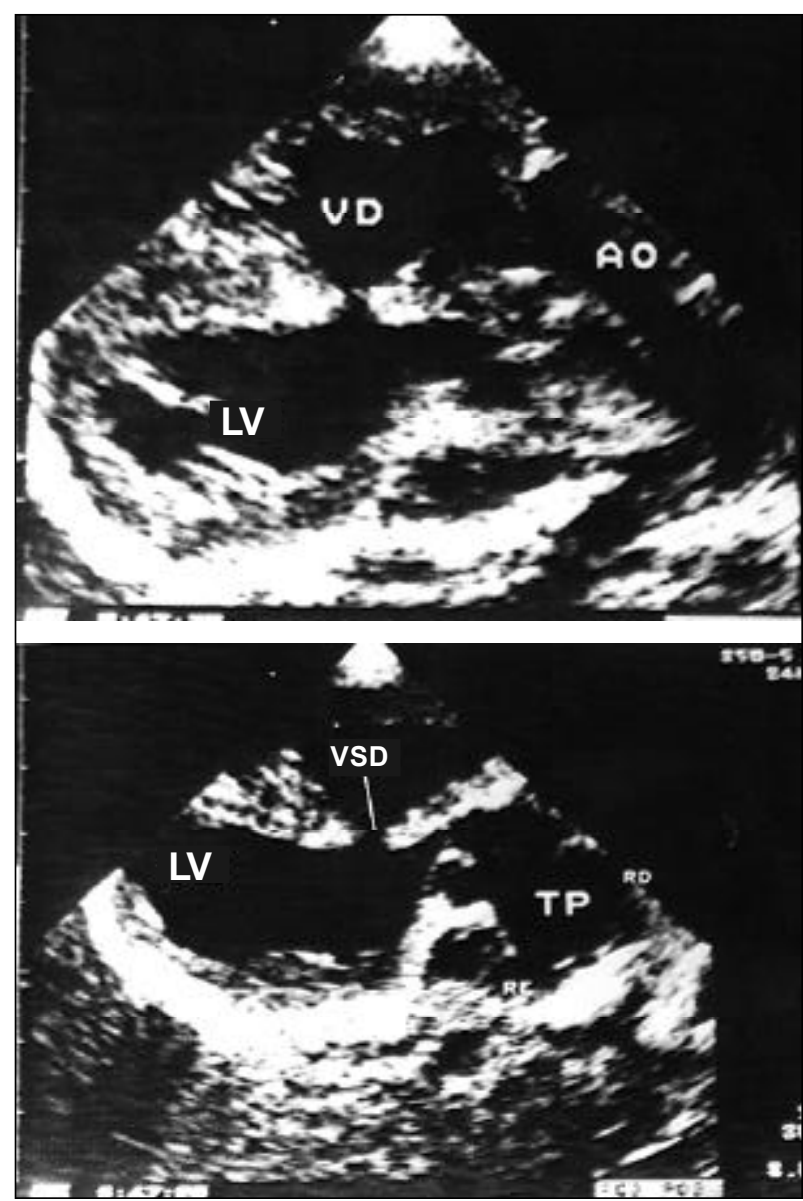

Fig. 3 - Longitudinal view, case 2. Transposition of the great arteries on echocardiography. Observe in the upper part, the aorta emerging from the right ventricle (RV), and, in the lower part, the pulmonary artery emerging from the left ventricle (LV). A ventricular septal defect may also be observed (VSD). RB - right branch of the pulmonary artery; LB - left branch of the pulmonary artery.

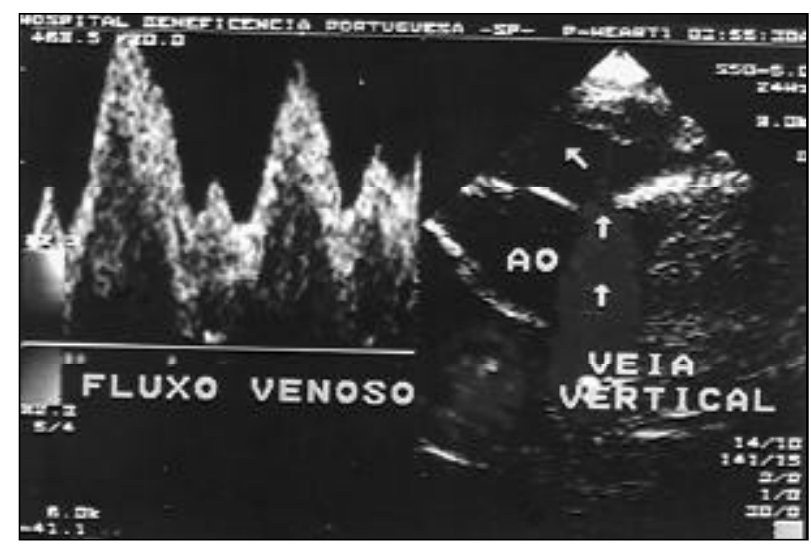

Fig. 4 - Echocardiogram, case 2. Echocardiographic diagnosis of partial anomalous pulmonary venous connection. Observe the blood flow in red in the vertical vein heading toward the innominate vein on the color-flow mapping (arrows). To the left, the confirmation of the venous flow pattern.

intensity of the cyanosis and the hemodynamic abnormalities caused by the transposition of the great arteries ${ }^{1,2,4}$. In this circumstance, the anomalous pulmonary venous connection in the right atrium avoids an increase in the pul- 
monary flow and enhances the saturation of the blood that reaches the aorta and the systemic circulation.

In the literature, a few authors ${ }^{1,2,4,5}$ document the surgical approach to this association reporting the successful repair in the atrial plane with the modified techniques by Senning or Mustard. One of the studies ${ }^{4}$ reports that the anatomical repair in the arterial plane, which is the first choice for the transposition of the great arteries isolated, is contraindicated when an association with total anomalous pulmonary venous connection exists, because the left ventricle is not prepared to receive the aorta surgically, due to its decreased flow situation since fetal life.

Analyzing the postoperative evolution of the 2 patients here reported, who underwent anatomical surgical repair, this theory seems not to be applicable, because unlike that which was expected, a good evolution existed in patient 1 , whose association was with the total form of anomalous pulmonary venous connection, and no sign of lack of left ventricular preparation or dysfunction occurred in the postoperative evolution. On the other hand, in patient 2 , in which a better result should occur according to this theory, because the left ventricle would be better adapted due to the partial form of the anomalous connection, death occurred on the $3^{\text {rd }}$ postoperative day.

Knowing that $4 \%$ of the newborn babies with transposition of the great arteries die rapidly before the anatomical surgical repair, Maeno et $\mathrm{al}^{6}$ tried to establish, through fetal echocardiography, a group of high-risk fetuses with this congenital heart disease. The intrauterine search for echocardiographic signs of restriction of the oval foramen or ductus arteriosus would be much more important, as a prognostic factor, than the preparation of the left ventricle, which would lead to early neonatal death independent from the aggressive maneuvers of reanimation or early surgical intervention. Therefore, we think that the anatomical repair for transposition of the great arteries should be considered even when this malformation is associated with anomalous pulmonary venous connection.

In case 1, in which echocardiography failed to diagnose the association with the anomalous pulmonary venous connection, a new echocardiographic examination was performed after the cardiac catheterization, to check whether the diagnosis of the anomalous vein was possible or not with the aid of this noninvasive method. We observed that the anomalous vein was perfectly visible and that the diagnostic failure was probably caused by not considering the possibility of this association; this resulted in an inadequate analysis of the subcostal region, which was then restricted to the diagnosis of the situs. The significant dilation of the right chambers was also not considered in case 1 , which had much more exuberant findings than did case 2 , and could not be explained by only transposition of the great arteries (fig. 1).

Knowing how unexpected the morphological diagnosis of an examination in a patient with congenital heart disease may be, it is of fundamental importance to observe the sequence of the segmentary analysis, which should always be performed in pediatric echocardiography through logical systematics.

In conclusion, the echocardiographic diagnosis of transposition of the great arteries associated with anomalous pulmonary venous connection is possible. The severe dilation of the right chambers in a mildly cyanotic child with transposition of the great arteries seems to be the diagnostic clue to be remembered for this rare association.

\section{Acknowledgments}

We thank Drs. José Pedro da Silva and Gláucio Furlanetto, whose teams assisted clinically and surgically the patients reported.

\section{References}

1. Amadeo A, Corno A, Marino B, Carta MG, Marcelletti C. Combined repair of transposed great arteries and total anomalous pulmonary venous connection. Ann Thorac Surg 1990; 50: 820-1.

2. Barbero-Marcial M, Verginelli G, Vila J, Zerbini EJ. Transposition of the great arteries associated with total anomalous pulmonary venous connection: a surgical approach. Ann Thorac Surg 1984; 37: 92-4.

3. Delisle G, Ando M, Galder AL, et al. Total Anomalous pulmonary venous connection: report of 93 autopsied cases with emphasis on diagnostic and surgical considerations. Am Heart J 1976; 91 : 99.
4. Gontijo B, Fantini F, Barbosa M, Gomes VM, Gutierrez C, Vrandecic M. Surgical repair of transposition of great arteries and total anomalous pulmonary venous return of the coronary sinus. Eur J Cardio-Thorac Surg 1994; 8: 391-2.

5. Ueda Y, Miki S, Okita Y, et al Transposition of the great arteries associated with total anomalous pulmonary venous return. Ann Thorac Surg 1994; 57: 470-2.

6. Maeno YV, Kamenir AS, Sinclair B, Van der Velde ME, Smallhorn JF, Hornberger LK. Prenatal features of ductus arteriosus constriction and restrictive foramen ovale in d-transposition of the great arteries. Circulation 1999; 99:1209-14. 